\title{
Evaluation of Pentosidine as Bone Quality Marker in Postmenopausal Breast Cancer Patients Receiving Aromatase Inhibitors
}

Hideo Shigematsu ( $\square$ shigematu1330@yahoo.co.jp )

kure medical center and chugoku cancer center https://orcid.org/0000-0001-9393-9655

Tomoyuki Yoshiyama

kure medical center and chugoku cancer center

Daisuke Yasui

kure medical center and chugoku cancer center

Shinji Ozaki

hiroshima prefectural hospital

Research article

Keywords: serum pentosidine, breast cancer, aromatase inhibitor, bone modifying agents

Posted Date: July 24th, 2020

DOI: https://doi.org/10.21203/rs.3.rs-42565/v1

License: (c) (i) This work is licensed under a Creative Commons Attribution 4.0 International License.

Read Full License 


\section{Abstract}

Background: Osteoporosis and fractures are important aromatase inhibitor (Al) related adverse events in postmenopausal women with hormone receptor positive breast cancer. An increment of pentosidine is associated with a deterioration of bone quality. In this study, pentosidine was evaluated in postmenopausal breast cancer patients receiving Als.

Methods: Fifty postmenopausal breast cancer patients receiving Als were retrospectively evaluated. Sixteen patients were given a bone modifying agent (BMA) concomitant with Als. Changes of pentosidine, bone turnover markers and bone mineral density (BMD) before and after 12 months of $\mathrm{Al}$ therapy were compared between BMA administered patients (BMA group) and a non-BMA group.

Results: There was no significant difference between pentosidine low and high groups in regard to age, height, weight, BMD of femoral neck and lumbar spine, and bone turnover markers including TRACP-5b and BAP. In the non-BMA group, pentosidine was increased in 18 cases (53\%), and the average change of pentosidine was $21.5 \%$ ( $95 \% \mathrm{Cl} ; 0.23$ to $42.7 \%, \mathrm{p}=0.048)$. In the BMA group, pentosidine was increased in 2 two cases (13\%), and the average change of pentosidine was $-16.6 \%(95 \% \mathrm{Cl} ;-30.6$ to $-2.6 \%, p=0.023)$. There was a significantly lower proportion of pentosidine-increased cases $(p=0.0065)$ and decrease of pentosidine $(p=0.021)$ in the BMA group compared to those in the non-BMA group.

Conclusions: Pentosidine was an independent factor from risk factors of osteoporotic fracture. Pentosidine was increased with Al, however, BMA inhibits an Al-induced increase of pentosidine in postmenopausal breast cancer patients.

\section{Background}

More than $70 \%$ of early breast cancers are hormone-receptor positive, and adjuvant endocrine therapy reduces breast cancer recurrence and improves overall survival in patients with hormone receptor positive breast cancer[1]. Administration of an aromatase inhibitor (Al) for 5-10 years has become standard therapy for postmenopausal woman with hormone receptor positive breast cancer[2]. Al inhibits the peripheral conversion of androgens to estrogens, which leads to estrogen deficiency resulting in bone loss and vulnerability. Compared with tamoxifen, a selective estrogen receptor modulator, the long-term administration of $\mathrm{Al}$ is associated with a higher incidence of osteoporosis and bone fracture[3]. In addition to bone density, bone quality is regarded as an independent factor of bone strength[4]. Bone quality is determined by bone collagen and collagen crosslinks which determines biomechanical structures and pliability[5]. Collagen cross-links are classified into physiological cross-links and advanced glycation end products (AGEs) cross-links, and hyperplasia of AGEs reduces suppleness that results in hard and brittle bone. Pentosidine is an AGE and hyperplasia of bone pentosidine is associated with a deterioration of bone strength without a decrement of BMD[6]. Several studies evaluated the association between osteoporotic fractures and urinary/serum level of pentosidine. In a multicenter cohort study of osteoporosis patients under bisphosphonate treatment, a higher baseline urinary pentosidine level is 
associated with development of vertebral fracture[7]. In a hospital-based cohort study of postmenopausal women, increment of urinary pentosidine were significantly associated with incidence of vertebral fracture in multivariate Cox regression analysis[8]. The serum pentosidine level is also associated with deteriorated bone strength and incidence of vertebral fracture $[9,10]$. In a cohort study of patients with prostate cancer undergoing androgen deprivation therapy, serum pentosidine was increased after endocrine therapy and denosumab inhibited the increment of serum pentosidine[11]. However, there are no reports of pentosidine in breast cancer patients receiving Al therapy and the effect of a bone modifying agent (BMA) on changes from pentosidine is uncertain.

In this study, pentosidine in addition to BMD and a bone turnover marker were evaluated retrospectively in postmenopausal breast cancer patients receiving Als with or without BMAs.

\section{Materials And Methods}

\section{Subjects}

A retrospective evaluation was done for a total of 50 consecutive postmenopausal patients that were administered Als as adjuvant therapy for hormone receptor positive early breast cancer at Kure Medical Center and the Chugoku Cancer Center, Kure, Japan, between October 2016 and December 2019. All patients received steroidal Als, anastrozole or letrozole, as adjuvant endocrine therapy. BMAs including bisphosphonate or denosumab were administered for 16 patients who were diagnosed as having osteoporosis or high risk for fracture based on BMD and general condition. Osteoporosis was determined as a T-score less than -2.5 at either the lumbar spine or total hip. Patients with a T-score greater than 2.5 but less than -1.0 at either the lumbar spine or total hip and with clinical risk factors for fracture were also determined high risk for fracture. Among these patients, 10 received denosumab $60 \mathrm{mg}$ every six months, while six received bisphosphonate. Prescribed bisphosphonate was oral sodium risedronate hydrate in two, oral alendronate sodium hydrate in two, and intravenous zoledronic acid in two patients. Changes of pentosidine, bone turnover markers and BMD before and after 12 months of Al therapy were compared between BMA administered patients (BMA group) and a non-BMA group. Patients with a history of fracture, chronic renal failure or diabetes requiring insulin therapy were excluded from this study.

\section{Measurement of pentosidine, bone turnover marker and BMD}

Pentosidine and bone turnover markers, including TRACP-5b and BAP, were evaluated using blood samples. Fasting blood samples were collected before and 12 months after administration of Al and were stored at $4{ }^{\circ} \mathrm{C}$ for measurement of BAP and $-80^{\circ} \mathrm{C}$ for measurement of TRACP-5b and pentosidine. TRACP-5b was measured using enzyme immunoassays by Osteolinks enzyme immunoassays (SB bioscience). Intra-assay CV was less than $15 \%$. Bone alkaline phosphatase (BAP) was measured by 
Access Ostase immunoassay (Beckman Coulter). Intra-assay CV was 7.4\%. Pentosidine was measured using enzyme-linked immunosorbent assays by microplate reader Bench mark (SAKURA). Intra-assay CV was $5.5 \%$. BMD was measured in the lumbar spine and femoral neck before and 12 months after administration of Al using dual-energy X-ray (DXA) absorptiometry with the Horizon DXA system (Hologic, Japan, Inc). The quality control was performed using a spine phantom. Intra-assay CV was $0.6 \%$.

\section{Statistical analysis}

A chi-square test was used to compare the proportion of patients between the BMA and non-BMA group. Patient characteristics and changes in bone markers and BMD between the BMA and non-BMA group were analyzed with Student's t-tests. A regression analysis was used to evaluate the relation between changes of pentosidine and bone turn over markers. Statistical analyses were performed using SPSS software (version 11 for Windows; 5 SAS Institute, Tokyo, Japan). A P value $<0.05$ was considered as statistically significant.

\section{Results}

\section{Relationship between serum pentosidine and clinical factors}

The median age of the subjects was 67 years, and 21, 23 and 6 subjects were classified as normal, bone loss and osteoporosis, respectively, according to the BMD results. Table 1 shows the relationship between pentosidine and other clinical factors. The subjects were classified into a pentosidine high or pentosidine low group according to the median value of pentosidine $0.555 \mu \mathrm{g} / \mathrm{ml}$. There was no significant difference between the pentosidine high and pentosidine low group in regard to age, height, weight, BMD of the lumber spine and femoral neck and bone turnover markers including TRACP-5b and BAP. No patient experienced breast cancer recurrence, non-traumatic fractures or osteonecrosis of the jaw during this study period. 
Table 1

Clinical factors of patients according to administration of BMA

\begin{tabular}{|llll|}
\hline Factors & $\begin{array}{l}\text { Pentosidine low } \\
(\mathbf{n = 2 5})\end{array}$ & $\begin{array}{l}\text { Pentosidine high } \\
(\mathbf{n}=\mathbf{2 5})\end{array}$ & $\begin{array}{l}\text { p value } \\
(\mathbf{t} \text { test })\end{array}$ \\
\hline Age $($ mean \pm S.D. $)$ & $66.6 \pm 1.6$ & $66.1 \pm 1.6$ & 0.83 \\
\hline Height $(\mathrm{cm}$, mean \pm S.D. $)$ & $154.2 \pm 1.1$ & $153.8 \pm 1.1$ & 0.83 \\
\hline Weight $(\mathrm{kg}$, mean \pm S.D. $)$ & $60.6 \pm 2.2$ & $60.5 \pm 2.2$ & 0.98 \\
\hline BMD of lumber spine $(\mathrm{g} / \mathrm{cm} 3$, mean \pm S.D.) & $0.885 \pm 0.030$ & $0.890 \pm 0.030$ & 0.9 \\
\hline BMD of femoral neck $(\mathrm{g} / \mathrm{cm} 3$, mean \pm S.D. $)$ & $0.745 \pm 0.025$ & $0.773 \pm 0.025$ & 0.42 \\
\hline TRACP-5b (mU/dl, mean \pm S.D. $)$ & $501.4 \pm 31.3$ & $478.2 \pm 31.3$ & 0.6 \\
\hline BAP $(\mu \mathrm{g} / \mathrm{l}$, mean \pm S.D. $)$ & $16.0 \pm 1.7$ & $16.8 \pm 1.7$ & 0.73 \\
\hline S.D., Standard deviation; BMD, bone mineral density; BMA, bone modifying agent & \\
\hline
\end{tabular}

\section{Changes in serum pentosidine according to BMA}

Table 2 shows the change of pentosidine in the BMA- group and BMA + group. In the non-BMA group, pentosidine increased in 18 cases (53\%), and the average change of pentosidine was $21.5 \%(95 \% \mathrm{Cl} ; 0.23$ to $42.7 \%, p=0.048)$. In the BMA group, pentosidine increased in two cases $(13 \%)$, and the average change of pentosidine was $-16.6 \%(95 \% \mathrm{Cl} ;-30.6$ to $-2.6 \%, p=0.023)$. A significantly lower proportion of pentosidine-increased cases $(p=0.0065)$ and decrease of pentosidine $(p=0.021)$ were recognized in the BMA group compared with the non-BMA group. 
Table 2

A. Proportion of patients with an increase or decrease of pentosidine from baseline to 12 months according to BMA administration. B. Percentage change from baseline to 12 months in pentosidine according to BMA administration.

\begin{tabular}{|llll|}
\hline Table 2A & & \\
\hline Pentosidine & BMA- & BMA+ & p value (Pearson) \\
\hline Increase & 18 & 14 & 0.0065 \\
\hline Decrease & 16 & \\
\hline Table 2B & & \\
& Change from baseline (\%, mean \pm S.D. $)$ & p value (t test) \\
BMA- & $21.5(0.23$ to 42.7$)$ & 0.021 \\
\hline BMA+ & $-16.6(-30.6$ to -2.6$)$ & \\
\hline S.D., Standard deviation; BMA, bone modifying agent \\
\hline
\end{tabular}

\section{Changes in bone turnover markers and BMD according to BMA}

Table 3 shows changes in bone turnover markers and BMD in the femoral neck and lumbar spine. After 12 months treatment with Als, the mean changes of TRACP-5b $(-54.4 \%$ vs. $10.9 \%, p<0.001)$ and BAP $(-$ $44.8 \%$ vs. $19.3 \%, p<0.001$ ) were significantly lower in the BMD + group compared with BMA-group, respectively. In regard to $B M D$, the mean changes of femoral neck $(3.9 \%$ vs. $-1.9 \%, p<0.001)$ and lumbar spine $(7.4 \%$ vs. $-0.93 \%, p<0.001)$ were significantly higher after 12 months of treatment with Als in the $\mathrm{BMD}+$ group compared with the BMA-group, respectively. The association between changes of pentosidine and bone turnover markers, including TRACP-5b and BAP was evaluated using regression analysis. Neither change of TRACP-5b $\left(R^{2}=0.027, p=0.25\right)$ nor BAP $\left(R^{2}=0.026, p=0.26\right)$ was significantly associated with change of pentosidine. 
Table 3

Percentage change from baseline to 12 months in BMD and bone turnover markers according to BMA administration

\begin{tabular}{|c|c|c|c|}
\hline & & $\begin{array}{l}\text { Change from baseline } \\
(\%, \text { mean } \pm \text { S.D. })\end{array}$ & $\begin{array}{l}\mathrm{p} \text { value } \\
\text { ( } \mathrm{t} \text { test) }\end{array}$ \\
\hline \multirow[t]{2}{*}{ BMD of lumber spine $(\mathrm{g} / \mathrm{cm} 3$, mean \pm S.D. $)$} & BMA- & $-0.93 \pm 0.76$ & $<0.001$ \\
\hline & BMA+ & $7.4 \pm 1.1$ & \\
\hline \multirow[t]{2}{*}{ BMD of femoral neck $(\mathrm{g} / \mathrm{cm} 3$, mean \pm S.D. $)$} & BMA- & $-1.9 \pm 0.60$ & $<0.001$ \\
\hline & BMA+ & $3.9 \pm 0.86$ & \\
\hline \multirow[t]{2}{*}{ TRACP-5b (mU/dl, mean \pm S.D.) } & BMA- & $10.9 \pm 3.3$ & $<0.001$ \\
\hline & BMA+ & $-54.4 \pm 4.8$ & \\
\hline \multirow[t]{2}{*}{ BAP $(\mu \mathrm{g} / \mathrm{l}$, mean \pm S.D. $)$} & BMA- & $19.3 \pm 4.6$ & $<0.001$ \\
\hline & BMA+ & $-44.8 \pm 6.6$ & \\
\hline
\end{tabular}

\section{Discussion}

In this cohort study of postmenopausal breast cancer patients receiving Als as adjuvant therapy, the bone quality marker pentosidine was increased by $\mathrm{Al}$ and reduced with BMAs. Bone turnover markers and BMD also showed significant improvement with BMAs. These results indicate that administration of BMA exerts not only an inhibition of bone loss, but also an improvement of bone quality in patients receiving Als.

Fracture and osteoporosis are important adverse events in postmenopausal breast cancer patients receiving adjuvant Als, and the prophylactic administration of BMA is recommended for patients with a high risk of bone-related adverse events[12, 13]. Because long-term prescription of BMA can cause serious adverse events, such as osteonecrosis of the jaw and atypical femoral fracture in breast cancer, an appropriate evaluation of the risk of $\mathrm{Al}$ related fracture is desirable[14, 15]. Although low BMD in femoral neck or lumbar spine is regarded as an independent risk factor for fracture, a certain proportion of patients with a non-osteoporotic range of BMD experience nontraumatic fractures[16, 17]. In a cohort study of postmenopausal women, more than half of osteoporotic fractures occur in cases with a nonosteoporotic range of BMD[18]. The Fracture Risk Assessment Tool (FRAX) is used to estimate the risk of osteoporotic fracture using femoral neck BMD and clinical risk factors, which include age, prior nontraumatic fracture, glucocorticoid use, low body mass index, family history of osteoporosis, smoking, and excess alcohol intake, which are also associated with incidence of fracture. However, FRAX is not designed to estimate the risk of Al-related fracture, and the estimated fracture risk by FRAX tends to be lower than that of the actual fracture incidence in breast cancer patients receiving AI[19]. Thus, the 
evaluation of other risk factors is warranted to determine appropriate administration of BMA to prevent Al-related fracture in postmenopausal breast cancer patients.

Independent of BMD, bone quality is regarded as an important factor of bone strength when considering the risk of osteoporotic fracture. The trabecular structure, including trabecular number, separation and homogeneity of the trabecular network, is also deteriorated with exemestane. A follow-up study of 29,407 postmenopausal women aged 50 years and older showed that patients with bone loss by BMD and a low Trabecular Bone Score (TBS) were at the same risk of fracture as those with osteoporosis by BMD and high TBS[20]. In an accompanying study of a randomized control trial evaluating exemestane, an Al, for prevention of breast cancer in healthy postmenopausal women, high-resolution peripheral quantitative CT showed a significant decrease in volumetric BMD and cortical thickness at the distal radius and distal tibia with exemestane[21]. These findings suggest that deterioration of bone quality is also an important Al-related bone adverse effect.

Serum and urine pentosidine have been investigated as bone quality markers. Pentosidine is positively correlated with deteriorated collagen crosslinks AGEs[6], and elevated pentosidine is shown as an independent risk factor of fracture in postmenopausal women[22]. In this study, we evaluated the clinical significance of pentosidine in postmenopausal breast cancer patients receiving adjuvant Als. First, pentosidine was shown to be independent from other risk factors of osteoporotic fracture. When patients were divided into a pentosidine low and high group according to median value of pentosidine, there was no significant difference between the two groups in regard to age, height, weight, BMD of lumber spine and femoral neck and bone turnover markers including TRACP-5b and BAP. This finding indicates that measurements of pentosidine may lead to a more accurate assessment of fracture risk based on conventional BMD and physical factors. Second, approximately half of subjects who were administered adjuvant Als had an elevation of pentosidine. Al treatment is associated with not only a decrease of BMD, but also a deterioration of bone quality. Finally, in this study, the administration of BMA significantly suppressed an Al-induced pentosidine increase and BMD decrease. Our findings are consistent with a previous study evaluating the effects of denosumab on prostate cancer patients treated with hormonal therapy. BMA therapy can maintain BMD and prevent a deterioration of bone quality in patients treated with hormonal therapy.

This study has several limitations. First, this is a cohort study with a relatively small number of cases and a short duration of follow up. A prospective study with a larger number of cases with a long-term period is warranted to confirm our findings. Second, BMAs were essentially prescribed for patients with a high risk of osteoporotic fracture determined by low BMD and/or clinical factors in this study. The effect of BMA on preventing Al-induced bone quality deterioration is uncertain in patients with a normal range of BMD. Finally, no bone quality assessment other than pentosidine was done in this study. Imaging studies evaluating bone structure should be done in future study to confirm our findings.

In summary, this study demonstrated that serum pentosidine significantly increased with Al therapy in postmenopausal women with hormone receptor positive breast cancer, and administration of BMAs 
inhibit an Al-induced increase of pentosidine. A prospective long-term study is warranted to confirm the relationship between pentosidine and non-traumatic fracture during adjuvant Al therapy.

\section{Abbreviations}

Al: aromatase inhibitor; BMA: bone modifying agent; AGEs: advanced glycation end products; BMD: bone mineral density; DXA: dual-energy X-ray; S.D: Standard deviation

\section{Declarations}

\section{Acknowledgements}

None.

\section{Authors' contributions}

HS: construction of this clinical study, data collection and writing the original manuscript. TY: data collection and editing manuscript. DY: data collection and editing manuscript. SO: designing the study, and reviewing and editing the manuscript. All authors read and approved the final manuscript.

\section{Funding}

There was no grant for this study.

\section{Availability of data and materials}

The data analyzed in this study are available from the corresponding author on appropriate reason.

\section{Ethics approval}

This study was approved by the Kure Medical Center ethics review board (29-70).

\section{Consent for publication}

Not applicable.

\section{Competing interests}

All authors have no conflicts of interest in this study. 


\section{References}

1. Early Breast Cancer Trialists' Collaborative G (2005) Effects of chemotherapy and hormonal therapy for early breast cancer on recurrence and 15-year survival: an overview of the randomised trials. Lancet 365:1687-717 doi:10.1016/S0140-6736(05)66544-0

2. Early Breast Cancer Trialists' Collaborative G (2015) Aromatase inhibitors versus tamoxifen in early breast cancer: patient-level meta-analysis of the randomised trials. Lancet 386:1341-52 doi:10.1016/S0140-6736(15)61074-1

3. Amir E, Seruga B, Niraula S, Carlsson L, Ocana A (2011) Toxicity of adjuvant endocrine therapy in postmenopausal breast cancer patients: a systematic review and meta-analysis. Journal of the National Cancer Institute 103:1299-309 doi:10.1093/jnci/djr242

4. Nih Consensus Development Panel on Osteoporosis Prevention D, Therapy (2001) Osteoporosis prevention, diagnosis, and therapy. Jama 285:785-95 doi:10.1001/jama.285.6.785

5. Saito M, Soshi S, Tanaka T, Fujii K (2004) Intensity-related differences in collagen post-translational modification in MC3T3-E1 osteoblasts after exposure to low- and high-intensity pulsed ultrasound. Bone 35:644-55 doi:10.1016/j.bone.2004.04.024

6. Saito M, Marumo K (2010) Collagen cross-links as a determinant of bone quality: a possible explanation for bone fragility in aging, osteoporosis, and diabetes mellitus. Osteoporosis international : a journal established as result of cooperation between the European Foundation for Osteoporosis and the National Osteoporosis Foundation of the USA 21:195-214 doi:10.1007/s00198-009-1066-z

7. Shiraki M, Kuroda T, Shiraki Y, Tanaka S, Higuchi T, Saito M (2011) Urinary pentosidine and plasma homocysteine levels at baseline predict future fractures in osteoporosis patients under bisphosphonate treatment. Journal of bone and mineral metabolism 29:62-70 doi:10.1007/s00774010-0191-2

8. Tanaka S, Kuroda T, Saito M, Shiraki M (2011) Urinary pentosidine improves risk classification using fracture risk assessment tools for postmenopausal women. Journal of bone and mineral research : the official journal of the American Society for Bone and Mineral Research 26:2778-84 doi:10.1002/jbmr.467

9. Yamamoto M, Yamaguchi T, Yamauchi M, Yano S, Sugimoto T (2008) Serum pentosidine levels are positively associated with the presence of vertebral fractures in postmenopausal women with type 2 diabetes. The Journal of clinical endocrinology and metabolism 93:1013-9 doi:10.1210/jc.20071270

10. Shiraki M, Kuroda T, Tanaka S, Saito M, Fukunaga M, Nakamura T (2008) Nonenzymatic collagen cross-links induced by glycoxidation (pentosidine) predicts vertebral fractures. Journal of bone and mineral metabolism 26:93-100 doi:10.1007/s00774-007-0784-6

11. Miyazawa Y, Sekine Y, Syuto T, Nomura M, Koike H, Matsui H, Shibata Y, Ito K, Suzuki K (2017) Evaluation of Bone Turnover / Quality Markers and Bone Mineral Density in Prostate Cancer Patients 
Receiving Androgen Deprivation Therapy with or without Denosumab. Anticancer research 37:366771 doi:10.21873/anticanres.11737

12. Bone HG, Wagman RB, Brandi ML, Brown JP, Chapurlat R et al. (2017) 10 years of denosumab treatment in postmenopausal women with osteoporosis: results from the phase 3 randomised FREEDOM trial and open-label extension. The lancet Diabetes \& endocrinology 5:513-23 doi:10.1016/S2213-8587(17)30138-9

13. Van Poznak C, Hannon RA, Mackey JR, Campone M, Apffelstaedt JP, Clack G, Barlow D, Makris A, Eastell R (2010) Prevention of aromatase inhibitor-induced bone loss using risedronate: the SABRE trial. Journal of clinical oncology : official journal of the American Society of Clinical Oncology 28:967-75 doi:10.1200/JC0.2009.24.5902

14. Kyrgidis A, Triaridis S, Vahtsevanos K, Antoniades K (2009) Osteonecrosis of the jaw and bisphosphonate use in breast cancer patients. Expert review of anticancer therapy 9:1125-34 doi:10.1586/era.09.74

15. Takahashi M, Ozaki Y, Kizawa R, Masuda J, Sakamaki K, Kinowaki K, Umezu T, Kondoh C, Tanabe Y, Tamura N, Miura Y, Shigekawa T, Kawabata H, Baba N, Iguchi H, Takano T (2019) Atypical femoral fracture in patients with bone metastasis receiving denosumab therapy: a retrospective study and systematic review. BMC cancer 19:980 doi:10.1186/s12885-019-6236-6

16. Siris ES, Genant HK, Laster AJ, Chen P, Misurski DA, Krege JH (2007) Enhanced prediction of fracture risk combining vertebral fracture status and BMD. Osteoporosis international : a journal established as result of cooperation between the European Foundation for Osteoporosis and the National Osteoporosis Foundation of the USA 18:761-70 doi:10.1007/s00198-006-0306-8

17. Nguyen ND, Eisman JA, Center JR, Nguyen TV (2007) Risk factors for fracture in nonosteoporotic men and women. The Journal of clinical endocrinology and metabolism 92:955-62 doi:10.1210/jc.2006-1476

18. Siris ES, Chen YT, Abbott TA, Barrett-Connor E, Miller PD, Wehren LE, Berger ML (2004) Bone mineral density thresholds for pharmacological intervention to prevent fractures. Archives of internal medicine 164:1108-12 doi:10.1001/archinte.164.10.1108

19. Leslie WD, Morin SN, Lix LM, Niraula S, McCloskey EV, Johansson H, Harvey NC, Kanis JA (2019) Performance of FRAX in Women with Breast Cancer Initiating Aromatase Inhibitor Therapy: A Registry-Based Cohort Study. Journal of bone and mineral research : the official journal of the American Society for Bone and Mineral Research 34:1428-35 doi:10.1002/jbmr.3726

20. Hans D, Goertzen AL, Krieg MA, Leslie WD (2011) Bone microarchitecture assessed by TBS predicts osteoporotic fractures independent of bone density: the Manitoba study. Journal of bone and mineral research : the official journal of the American Society for Bone and Mineral Research 26:2762-9 doi:10.1002/jbmr.499

21. Cheung AM, Tile L, Cardew S, Pruthi S, Robbins J, Tomlinson G, Kapral MK, Khosla S, Majumdar S, Erlandson M, Scher J, Hu H, Demaras A, Lickley L, Bordeleau L, Elser C, Ingle J, Richardson H, Goss PE (2012) Bone density and structure in healthy postmenopausal women treated with exemestane 
for the primary prevention of breast cancer: a nested substudy of the MAP.3 randomised controlled trial. The Lancet Oncology 13:275-84 doi:10.1016/S1470-2045(11)70389-8

22. Shiraki M, Kashiwabara S, Imai T, Tanaka S, Saito M (2019) The association of urinary pentosidine levels with the prevalence of osteoporotic fractures in postmenopausal women. Journal of bone and mineral metabolism 37:1067-74 doi:10.1007/s00774-019-01017-9 